\title{
Drug resistance among tuberculosis cases in the European Union and European Economic Area, 2007 to 2012
}

M J van der Werf (marieke.vanderwerf@ecdc.europa.eu) ${ }^{1}$, C Ködmö̈n ${ }^{1}$, V Hollo $^{1}$, A Sandgren ${ }^{1}$, P Zucs ${ }^{1}$

1. European Centre for Disease Prevention and Control (ECDC), Stockholm, Sweden

Citation style for this article:

van der Werf MJ, Ködmön C, Hollo V, Sandgren A, Zucs P. Drug resistance among tuberculosis cases in the European Union and European Economic Area, 2007 to 2012. Euro Surveill. 2014;19(10):pii=20733. Available online: http://www.eurosurveillance.org/ViewArticle.aspx?Articleld=20733

Article submitted on 04 November 2013 / published on 13 March 2014

The European Union and European Economic Area (EU/ EEA) tuberculosis (TB) surveillance system collects detailed information on resistance to TB drugs. Using this information, we provide an overview of the current TB drug resistance situation and trends in the EU/EEA by performing a descriptive analysis, including analysis of treatment outcomes, of the TB cases reported between January 2007 and December 2012. The percentages of TB cases with different drug resistance patterns have been stable with about $90 \%$ of the new laboratory-confirmed cases pan-susceptible, $6 \%$ monodrug-resistant, $2 \%$ polydrug-resistant, $2 \%$ multidrug drug-resistant (MDR) TB - excluding extensively drugresistant (XDR) TB -, and $0.2 \%$ XDR-TB. In previously treated laboratory-confirmed TB cases, the percentage with MDR-TB excluding XDR-TB declined until 2010 to $16 \%$ and remained stable thereafter. During the study period, the percentages of cases with monodrug- and polydrug-resistant TB remained constant at about $8 \%$ and $2 \%$ whereas the percentage of XDR-TB cases increased slightly to $2.6 \%$. Treatment outcome results for all cases have been stable with overall $77.9 \%$ of the pan-susceptible cases, $69.6 \%$ of the monoresistant cases, $68.2 \%$ of the polyresistant cases, $32.2 \%$ of the MDR-TB cases (excluding XDR-TB), and $19.1 \%$ of the XDR-TB cases treated successfully. The treatment success rate target for new pulmonary culture-positive MDR-TB cases of $70 \%$ has not been reached. In addition, drug resistance surveillance can be improved by more complete reporting of drug susceptibility results and treatment outcome.

\section{Introduction}

Anti-tuberculosis (TB) drug resistance is a major public health challenge. Patients infected with Mycobacterium tuberculosis bacilli resistant to TB drugs often require longer, expensive treatment regimens, and show poorer treatment outcomes. In 2011, the global incidence of TB was estimated to be 125 cases per 100,000 population, with about 12 million prevalent TB cases [1]. Of the prevalent cases, $630,000(5.3 \%)$ were estimated to have multidrug-resistant (MDR) TB [1]. The 53 countries of the World Health Organization (WHO) European Region notified 380,366 TB cases in 2011. For 127,936 (33.6\%), drug susceptibility testing (DST) results were available, and $29,473(19.0 \%)$ were diagnosed with MDR-TB. In the 29 reporting European Union (EU) and European Economic Area (EEA) countries the proportion of MDR-TB was $4.5 \%$, while in non EU/EEA countries the proportion was $25.6 \%$ [2]. Although information on MDR-TB and extensively drug-resistant (XDR) TB is systematically collected and reported by the WHO [1], less information is available on the burden of mono- and polydrug resistance, or any drug resistance.

In the EU/EEA, many countries have based their system for surveillance of anti-tuberculosis drug resistance on recommendations of a WHO and International Union Against Tuberculosis and Lung Disease (IUATLD) working group [3]. Starting with the 2007 TB cohort, TB surveillance data from the country level are reported annually to the European Surveillance System (TESSy) database, operated by the European Centre for Disease Prevention and Control (ECDC). The ECDC previously published an analysis of MDR and XDR-TB in the EU/ EEA using notification data from 2008 [4]. This analysis showed that MDR-TB remained a threat and that XDR-TB had been identified within the EU/EEA borders. In the annual European tuberculosis surveillance and monitoring reports, notifications on MDR-TB and XDR-TB are provided separately from data on resistance to isoniazid or rifampicin [2]. Although information on resistance to other TB drugs is collected in the TESSy database, this information is not routinely reported. Some EU countries or regions in EU countries have published detailed information on drug resistance to individual TB drugs [5-7]. For example, the United Kingdom reported an increase of the proportion of TB cases resistant to isoniazid from 1998 to 2005 [7]. In the same period, a study from Castilla y León, Spain, indicated that the incidence of primary drug resistance and monoresistance was low [8] and this information was used to establish a new standard anti-tuberculosis treatment. Systematic analysis of drug resistance data 
helps to identify strengths and remaining challenges in TB control as well as to guide actions. It can also be used to assess whether the targets set in the EU/ $E E A$, i.e. to test $100 \%$ of the culture-positive TB cases for resistance to first-line TB drugs, are achieved [9].

Analysis of characteristics of patients with drug resistance can help to identify populations most at risk. A study in London among individuals with isoniazid monoresistance showed that cases were more likely to be young adults, born in the United Kingdom and of white or black Caribbean ethnicity, imprisoned at the time of diagnosis, unemployed, drug dealers or sex workers [10]. Another study assessed risk factors for resistance to second-line anti-tuberculosis drugs in eight countries [11]. It showed that previous treatment with second-line drugs was the strongest risk factor, and resistance to fluoroquinolones and XDR-TB were more frequent in women than in men. In addition, unemployment, alcohol abuse, and smoking were associated with resistance to second-line injectable drugs. Although some risk factors for drug resistance are study-site-specific, others seem to be general.

To assess whether TB control programmes are able to provide adequate treatment and support, an analysis of TB treatment outcomes is useful. In general, cases infected with a TB strain that is resistant to TB drugs have a worse treatment outcome $[12,13]$. Other patient characteristics that are reported to be related to unsuccessful treatment outcomes are being male, older age, having pulmonary TB, alcohol dependence, homelessness, unemployment and diabetes $[12,14,15]$. In the TESSy database, only few patient characteristics are collected, which allows for a limited risk factor analysis.

An in-depth analysis of TB drug resistance in the EU/ EEA has not been performed. Therefore, we aim to provide an overview of the current TB drug resistance situation in the EU/EEA and its trend, characteristics of drug-resistant cases, and their treatment outcomes.

\section{Methods}

\section{Data source and collection}

Data were extracted from the TESSy database on 4 October 2013. Data from $27 \mathrm{EU}$ and EEA countries reporting DST results to ECDC were analysed. France, Italy, and Spain were not included as they are not reporting case-based drug resistance data to TESSy but report aggregated results to the World Health Organization's Tuberculosis Monitoring and Evaluation platform. DST data had been collected for the first line drugs ethambutol, isoniazid, rifampicin, and streptomycin, and for the second line drugs amikacin, capreomycin, ciprofloxacin, gatifloxacin, kanamycin, levofloxacin, moxifloxacin, and ofloxacin.
Data inclusion and surveillance definitions

Only confirmed TB cases according to the EU case definition [16] with data on drug susceptibility for at least isoniazid and rifampicin were analysed. Definitions and categories provided in the ECDC/WHO report on tuberculosis surveillance and monitoring in Europe 2013 were used [2]. 'Pan-susceptible' refers to a case susceptible to all drugs tested. 'Monodrug resistance' is defined as resistance to one anti-TB drug, while 'polydrug resistance' refers to resistance to two or more drugs, excluding MDR-TB. MDR-TB is defined as resistance to at least isoniazid and rifampicin. XDR-TB is a special form of MDR-TB defined as resistance to at least isoniazid and rifampicin with further resistance to a fluoroquinolone and a second-line injectable agent (amikacin, kanamycin or capreomycin). Any drug resistance refers to a case with resistance to at least one TB drug. The percentage tested for susceptibility to second-line drugs (injectable agents: amikacin, capreomycin, kanamycin; fluoroquinolones: ciprofloxacin, gatifloxacin, levofloxacin, moxifloxacin, and ofloxacin) was calculated for cases for whom DST results were reported for at least one fluoroquinolone and one injectable drug. 'Previously treated TB case' means that the case has received TB treatment before the current TB episode.

If data on previous treatment were not available, information on previous TB diagnosis was used. Treatment success is defined as a treatment outcome reported as 'cured' or 'completed' within 12 months after diagnosis in non-M(X)DR-TB cases, within 24 months in MDR-TB cases and within 36 months in XDR-TB cases.

\section{Analysis}

We performed a descriptive analysis of surveillance data to assess the burden and trends of drug resistance among TB cases in EU/EEA countries between January 2007 and December 2012. We described cases with available resistance data by previous TB treatment history and resistance type over the years covered. We analysed different resistance types by sex, age-group, origin, human immunodeficiency virus (HIV) status, site of disease and treatment success. Treatment outcome after 12 months was analysed for pan-susceptible, monoresistant, and polyresistant cases notified between 2007 and 2011, treatment outcome after 24 months for MDR-TB cases notified between 2007 and 2010 and treatment outcome after 36 months for XDR-TB cases notified between 2007 and 2009. Fisher's exact tests were used to compare categorical data. Chi-squared test for trends was used to analyse changes over time of categorical data using the ptrend command in STATA. A p value of $<0.05$ was considered significant. All data analyses were performed using STATA 12.1 (StataCorp LP, Texas, USA). 
Notified laboratory-confirmed tuberculosis cases with reported testing results for the first-line TB drugs isoniazid (H) and rifampicin (R), and multidrug-resistant TB cases with reported testing results for second-line TB drug resistance, EU/EEA, 2007-2012

\begin{tabular}{|c|c|c|c|c|c|c|}
\hline Year & 2007 & 2008 & 2009 & 2010 & 2011 & 2012 \\
\hline $\begin{array}{l}\text { Number of countries reporting case-based data for } \\
\text { drug susceptibility for the first-line TB drugs } H \text { and } R\end{array}$ & $26^{\mathrm{a}, \mathrm{b}}$ & $25^{\mathrm{a}, \mathrm{b}}$ & $26^{a, b}$ & $26^{\mathrm{a}, \mathrm{b}}$ & $26^{\mathrm{a}, \mathrm{b}}$ & $25^{\mathrm{a}, \mathrm{b}}$ \\
\hline Number of notified laboratory-confirmed TB cases & 41,943 & 39,628 & 40,220 & 37,401 & 37,577 & 35,279 \\
\hline $\begin{array}{l}\text { Notified laboratory-confirmed TB cases with reported } \\
\text { testing results for } \mathrm{R} \text { and } \mathrm{H} \text { resistance } \mathrm{N}(\%)\end{array}$ & $\begin{array}{c}26,622 \\
(63.5)\end{array}$ & $\begin{array}{c}27,688 \\
(69.9)\end{array}$ & $\begin{array}{c}28,356 \\
(70.5)\end{array}$ & $\begin{array}{l}27,831 \\
(74.4)\end{array}$ & $\begin{array}{c}28,985 \\
(77.1)\end{array}$ & $\begin{array}{c}27,694 \\
(78.5)\end{array}$ \\
\hline Number of notified MDR-TB cases & 1,511 & 1,556 & 1,499 & 1,382 & 1,421 & 1,310 \\
\hline $\begin{array}{l}\text { Notified MDR-TB cases with reported testing results for } \\
\text { resistance to second-line TB drugs N (\%) }\end{array}$ & $\begin{array}{c}310 \\
(20.5)\end{array}$ & $\begin{array}{c}366 \\
(23.5)\end{array}$ & $\begin{array}{c}585 \\
(39.0)\end{array}$ & $\begin{array}{c}869 \\
(62.9)\end{array}$ & $\begin{array}{c}983 \\
(69.2)\end{array}$ & $\begin{array}{c}891 \\
(68.0) \\
\end{array}$ \\
\hline Number of notified XDR-TB cases & 76 & 90 & 66 & 115 & 143 & 128 \\
\hline
\end{tabular}

EU/EEA: European Union/European Economic Area; MDR: multidrug resistant; TB: tuberculosis; XDR: extensively drug resistant.

27 EU/EEA countries provided case-based data. France, Italy, and Spain do not report case-based drug susceptibility results to the European Surveillance System (TESSy) database but report aggregated results to the World Health Organization's Tuberculosis Monitoring and Evaluation platform.

a Greece is not included in 2007, 2008 and 2012.

b Liechtenstein is not included after 2008 .

\section{Results}

\section{Completeness of drug resistance testing reporting}

In 2007, 26 EU/EEA countries reported case-based rifampicin and isoniazid susceptibility testing results for $63.5 \%$ of all laboratory-confirmed TB cases, while 25 countries reported this data in 2012. Liechtenstein reported case-based data on DST for rifampicin and isoniazid only in 2007 and Greece only from 2009 to 2011 (Table 1). The percentage of laboratory-confirmed TB cases with testing results reported increased gradually from $63.5 \%(26,622 / 41,943)$ in 2007 to $78.5 \%$ $(27,694 / 35,279)$ in 2012 . Five countries reported results for rifampicin and isoniazid susceptibility testing for $100 \%$ of the laboratory-confirmed TB cases in 2012.

Reporting of DST results for first-line TB drugs for culture-positive non-MDR-TB increased between 2007 and 2012 (Table 2), both for new and previously treated TB cases.

Reporting of testing results of MDR-TB cases for resistance to second-line TB drugs increased steeply from $20.5 \%(320 / 1,511)$ of the notified MDR-TB cases in 2007 to $68.0 \%(891 / 1,310)$ in 2012 (Table 1). In 2012, ten countries reported DST results for more than $95 \%$ of all MDR-TB cases for second-line TB drugs, while the corresponding number was six countries in 2007. The testing percentage for second-line TB drugs was largely determined by the low percentage of MDR-TB cases for which a test result for second-line drugs was reported in Romania and the high number of MDR-TB cases reported in this country, i.e. $53.6 \%(284 / 530)$ in 2012. Germany (8/60), Ireland (1/5), and Poland (4/31) reported test results for second-line drugs for $<50 \%$ of the MDR-TB cases. However, these countries reported a lower number of cases and thus affected the EU/EEA second-line drug susceptibility percentage to a lesser extent.

DST results to the first-line drugs ethambutol and streptomycin were reported in respectively $84.5 \%$ $(1,107 / 1,310)$ and $82.9 \%(1,086 / 1,310)$ of the MDR-TB cases in 2012. This had increased from respectively $66.5 \%(1,005 / 1,511)$ and $64.2 \%(970 / 1,511)$ in 2007 . For the most frequently tested second-line drugs, kanamycin and ofloxacin, results were reported in $22.0 \%$ $(332 / 1,511)$ and $20.8 \%(315 / 1,511)$ of the MDR-TB cases in 2007 and in $64.6 \%(846 / 1,310)$ and $62.3 \%(816 / 1,310)$ in 2012. In 2012, $40.9 \%(536 / 1,310)$ of the MDR-TB cases were reported to be tested for amikacin resistance and $37.5 \%(491 / 1,310)$ for capreomycin resistance. Reporting on testing results for resistance to other second-line drugs was infrequent. DST results for the drugs gatifloxacin, levofloxacin, and moxifloxacin were only collected from 2013 onwards with updates for previous year data. None of the EU/EEA countries have reported susceptibility testing results for gatifloxacin or levofloxacin for the years 2007 to 2012.

\section{Drug resistance notification and trends}

In new laboratory-confirmed TB cases, the percentage of those with any resistance reported remained stable at around $10 \%$ (Figure $1 \mathrm{~A}$ ). Also, the percentages of new laboratory-confirmed TB cases with reported monoresistance, polyresistance, MDR-TB (excluding XDR-TB), and XDR-TB remained stable at about $6 \%, 2 \%$, $2 \%$, and $0.2 \%$ respectively. 
Resistance to first-line tuberculosis drugs in new and previously treated culture-positive non-MDR-TB cases by year, EU/ EEA, 2007-2012

\begin{tabular}{|c|c|c|c|c|c|c|c|c|c|}
\hline \multirow[b]{2}{*}{ Year } & \multirow{2}{*}{$\begin{array}{l}\text { All culture } \\
\text { positive } \\
\text { non-MDR-TB } \\
\text { cases }^{\mathrm{a}}\end{array}$} & \multicolumn{2}{|c|}{ Isoniazid } & \multicolumn{2}{|c|}{ Rifampicin } & \multicolumn{2}{|c|}{ Streptomycin } & \multicolumn{2}{|c|}{ Ethambutol } \\
\hline & & $\begin{array}{l}\text { Tested } \\
\text { N (\%) }\end{array}$ & $\begin{array}{c}R \\
N(\%)\end{array}$ & $\begin{array}{l}\text { Tested } \\
\text { N (\%) }\end{array}$ & $\begin{array}{c}R \\
N(\%)\end{array}$ & $\begin{array}{l}\text { Tested } \\
\mathrm{N}(\%)\end{array}$ & $\begin{array}{c}R \\
N(\%)\end{array}$ & $\begin{array}{l}\text { Tested } \\
\text { N (\%) }\end{array}$ & $\begin{array}{c}R \\
\text { N (\%) }\end{array}$ \\
\hline \multicolumn{10}{|c|}{ New culture-positive non-MDR-TB cases } \\
\hline 2007 & 38,904 & $20,407(52.5)$ & $1,166(5.7)$ & $20,407(52.5)$ & $115(0.6)$ & $12,933(33.2)$ & $784(6.1)$ & $18,378(47.2)$ & $135(0.7)$ \\
\hline 2008 & 37,104 & $21,034(56.7)$ & $1,199(5.7)$ & $21,034(56.7)$ & $87(0.4)$ & $12,528(33.8)$ & $757(6.0)$ & $18,321(49.4)$ & $143(0.8)$ \\
\hline 2009 & 36,826 & $21,689(58.9)$ & $1,128(5.2)$ & $21,689(58.9)$ & $91(0.4)$ & $13,183(35.8)$ & $681(5.2)$ & $18,250(49.6)$ & $84(0.5)$ \\
\hline 2010 & 35,468 & $21,571(60.8)$ & $1,185(5.5)$ & $21,571(60.8)$ & $78(0.4)$ & $14,801(41.7)$ & $879(5.9)$ & $17,989(50.7)$ & $93(0.5)$ \\
\hline 2011 & 35,441 & $22,955(64.8)$ & $1,250(5.4)$ & $22,955(64.8)$ & $77(0.3)$ & $16,190(45.7)$ & $929(5.7)$ & $19,030(53.7)$ & $97(0.5)$ \\
\hline 2012 & 33,182 & $22,061(66.5)$ & $1,125(5.1)$ & $22,061(66.5)$ & $89(0.4)$ & $15,894(47.9)$ & $874(5 \cdot 5)$ & $18,717(56.4)$ & $77(0.4)$ \\
\hline \multicolumn{10}{|c|}{ Previously treated culture-positive non-MDR-TB cases } \\
\hline 2007 & 7,119 & $3,129(44.0)$ & $370(11.8)$ & $3,129(44.0)$ & $78(2.5)$ & $1,570(22.1)$ & $140(8.9)$ & $1,926(27.1)$ & $39(2.0)$ \\
\hline 2008 & 6,618 & $3,616(54.6)$ & $315(8.7)$ & $3,616(54.6)$ & $54(1.5)$ & $1,509(22.8)$ & $137(9.1)$ & $1,950(29.5)$ & $42(2.2)$ \\
\hline 2009 & 6,714 & $3,872(57.7)$ & 352 (9.1) & $3,872(57.7)$ & $66(1.7)$ & $1,642(24.5)$ & $140(8.5)$ & 2,083 (31.0) & $41(2.0)$ \\
\hline 2010 & 6,256 & $3,671(58.7)$ & $348(9.5)$ & $3,671(58.7)$ & $60(1.6)$ & $1,706(27.3)$ & $153(9.0)$ & $1,925(30.8)$ & $39(2.0)$ \\
\hline 2011 & 5,695 & $3,469(60.9)$ & 315 (9.1) & $3,469(60.9)$ & $54(1.6)$ & $1,657(29.1)$ & $155(9.4)$ & $1,811(31.8)$ & $35(1.9)$ \\
\hline 2012 & 5,200 & $3,263(62.8)$ & $257(7.9)$ & $3,263(62.8)$ & $57(1.7)$ & $1,667(32.1)$ & $132(7.9)$ & $1,871(36.0)$ & $23(1.2)$ \\
\hline
\end{tabular}

EU/EEA: European Union/European Economic Area; non-MDR-TB: non multidrug-resistant tuberculosis; R: resistant; TB: tuberculosis.

27 EU/EEA countries provided case-based data. France, Italy, and Spain do not report case-based drug susceptibility results to the European Surveillance System (TESSy) database but report aggregated results to the World Health Organization's Tuberculosis Monitoring and Evaluation platform. Liechtenstein reported case-based data on drug susceptibility testing for rifampicin and isoniazid only in 2007 and Greece only from 2009 to 2011.

a Only TB cases with information available on previous treatment are included.

In previously treated laboratory-confirmed TB cases, the percentage with any resistance and with MDR(excluding XDR-TB) declined until 2010 (Figure 1B) and remained stable thereafter with about $30 \%$ of the previously treated cases showing any resistance. The percentage of cases with monoresistance and polyresistance did not change. The percentage of XDR-TB cases, increased from $1.4 \%$ in 2007 to $2.6 \%$ in 2012 (chi-squared (1) for trend=26.8, p<0.0001).

In 2012, the percentage of TB cases with any resistance varied considerably across EU/EEA countries with $3.3 \%$ $(6 / 181)$ showing any resistance in Slovakia and $37.7 \%$ (90/239) in Estonia (Figure 2).

The percentage of TB cases with reported rifampicin monoresistance ranged between $0 \%$ and $1.3 \%$ in 2012 in the different EU/EEA countries (Table 3). For isoniazid monoresistance the range was $0 \%$ to $6.6 \%$ and for MDR-TB $0 \%$ to $25 \cdot 5 \%$.

The total number of notified MDR-TB (including XDR-TB) cases decreased from 1,511 in 2007 to 1,310 in 2012 (Table 1). Liechtenstein did not report to TESSy in 2012, but reported o MDR TB cases in 2007. The number of new cases with MDR-TB (including XDR-TB) remained stable over the years, 516 in 2007 and 542 in 2012, whereas the number of MDR-TB (including XDR-TB) cases that were previously treated decreased from 972 in 2007 to 727 in 2012 (Table 4). The percentage of new MDR-TB (including XDR-TB) cases of MDR-TB cases for which previous treatment was known, increased from $34.7 \%(516 / 1,488)$ in 2007 to $(542 / 1,269) 42.7 \%$ in 2012 (chi-squared (1) test for trend=35.4, p<0.001).

Of the new MDR-TB (including XDR-TB) cases, $\geq 50 \%$ were reported to be resistant to ethambutol and $>80 \%$ to streptomycin (Table 4). Among previously treated MDR-TB (including XDR-TB) cases, around 70\% tested resistant to ethambutol, whereas resistance to streptomycin was comparable with the percentage in new MDR-TB cases. Resistance to kanamycin was reported in $40.7 \%$ of the new MDR-TB cases in 2007 and $23.5 \%$ in 2012. Ofloxacin resistance was less frequent in new MDR-TB cases with only $11.7 \%$ of the cases showing resistance in 2012. As expected, previously treated cases were more frequently resistant to both firstline and second-line TB drugs. Of all MDR-TB (including XDR-TB) cases tested for resistance to any of the second-line TB drugs between 2007 and 2012, 44.5\% $(1,782 / 4,004)$ were resistant to any of the second-line drugs. 


\section{FIGURE 1}

Resistance pattern among new (a) and previously treated (b) laboratory-confirmed TB cases tested for at least isoniazid and rifampicin resistance by year, EU/EEA, 2007-2012

A. New laboratory-confirmed TB cases

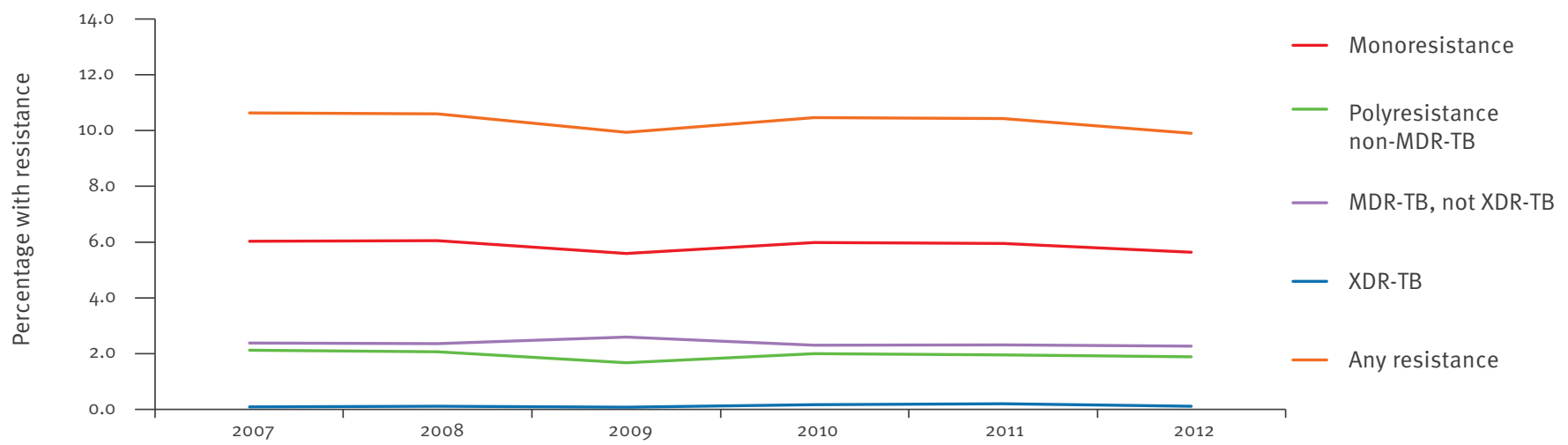

B. Previously treated laboratory-confirmed TB cases

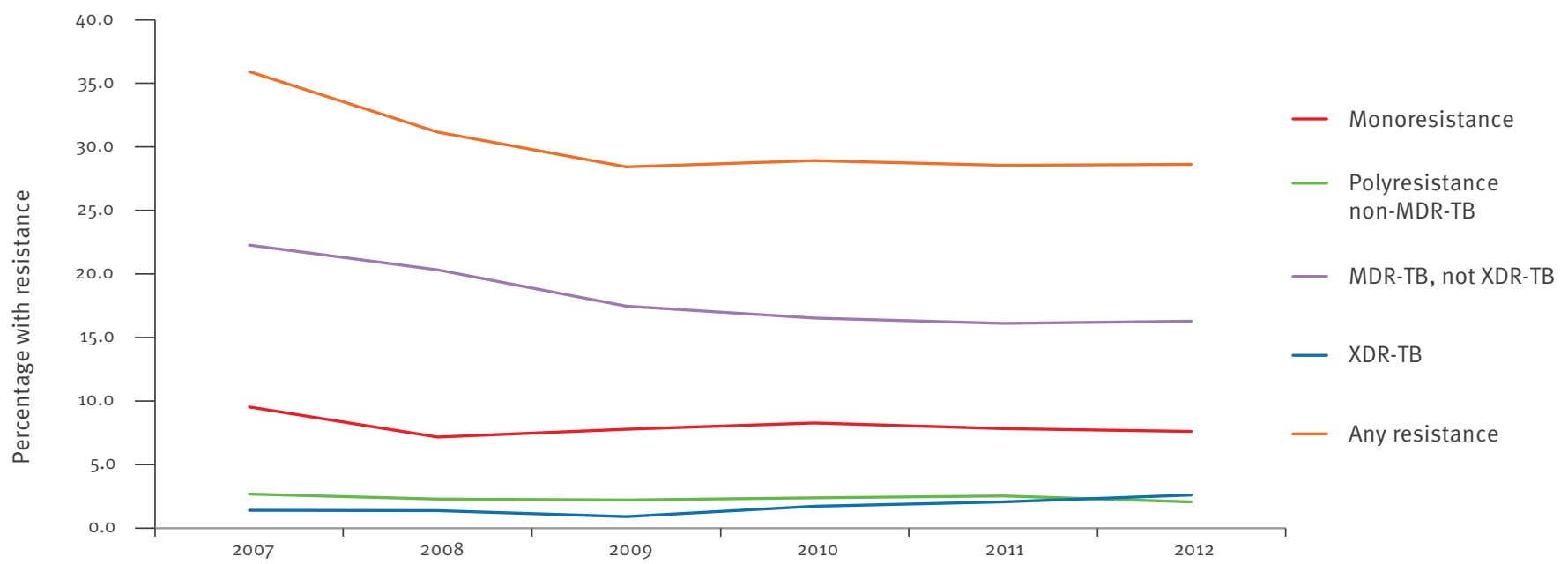

EU/EEA: European Union/European Economic Area; MDR-TB: multidrug-resistant tuberculosis; TB: tuberculosis; XDR-TB: extensively drug-resistant tuberculosis.

27 EU/EEA countries provided case-based data. France, Italy, and Spain do not report case-based drug susceptibility results to the European Surveillance System (TESSy) database but report aggregated results to the World Health Organization's Tuberculosis Monitoring and Evaluation platform. Of the 27 EU/EEA countries included in the study, Liechtenstein reported case-based data on drug susceptibility testing for rifampicin and isoniazid only in 2007 and Greece only from 2009 to 2011.

For 143 of the $183(78.1 \%)$ MDR-TB cases (including XDR-TB) with ciprofloxacin resistance, no cross resistance with other fluoroquinolones was reported. Of the 815 MDR-TB cases (including XDR-TB) with ofloxacin resistance, for $727(89.2 \%)$ no resistance to other fluoroquinolones was reported, and of the 55 cases resistant to moxifloxacin, for three $(5.5 \%)$ no other resistance to fluoroquinolones was reported. Thirty-six MDR-TB cases (including XDR-TB) were reported with resistance to both ciprofloxacin and ofloxacin and 48 showed resistance to both ofloxacin and moxifloxacin. Only four MDR-TB (including XDR-TB) cases were reported with resistance to all three fluoroquinolones.

\section{Characteristics of tuberculosis cases with drug resistance}

In $2012,32.6 \%$ of all laboratory-confirmed TB cases tested for at least isoniazid and rifampicin resistance were female (Table 5). In the groups with MDR-TB (excluding XDR-TB), and XDR-TB, females accounted for a lower proportion compared to the other groups, $25.6 \%$ and $21.9 \%$ of all cases, respectively ( $p<0.001$ and $p=0.008$, respectively). 


\section{FIGURE 2}

Percentage of laboratory-confirmed tuberculosis cases tested for at least isoniazid and rifampicin resistance with any drug resistance, EU/EEA, 2012
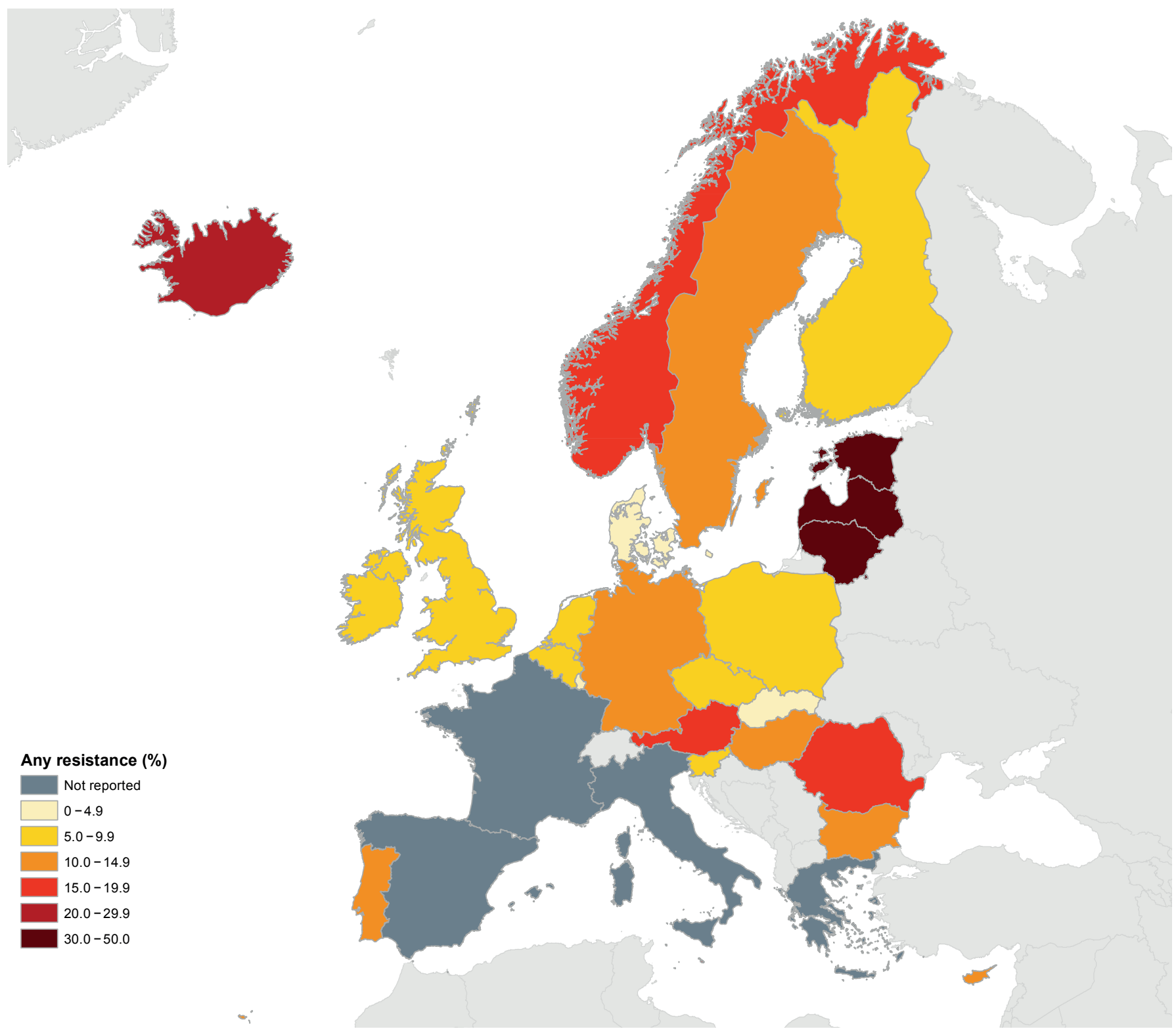

Any resistance (\%)

Not reported

$0-4.9$

$5.0-9.9$

$10.0-14.9$

$15.0-19.9$

$20.0-29.9$

$30.0-50.0$

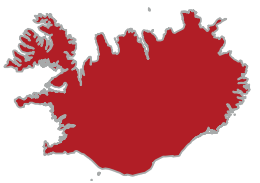

EU/EEA: European Union/European Economic Area.

The 'Not reported' legend indicates that case-based drug susceptibility results are not reported to the European Surveillance System (TESSy).

a France, Italy, and Spain do not report case-based drug susceptibility results to the European Surveillance System (TESSy) database but report aggregated results to the World Health Organization's Tuberculosis Monitoring and Evaluation platform. Liechtenstein and Greece did not report to TESSy in 2012.

Most notified TB cases were between 25 and 64 yearsold. The percentages of 25 to 64 year-olds were higher compared to those aged younger or older for XDR-TB (85.2\%) and MDR-TB (excluding XDR-TB) (82.2\%) cases when compared to pan-susceptible (70.1\%) or monoresistant $(74.4 \%)$ cases (comparison XDR-TB vs pan-susceptible, chi-squared (1)=13.8 p<0.001; comparison MDR-TB (excluding XDR-TB) vs pan-susceptible, chi-squared (1) =79.8 p<0.001; comparison XDR-TB vs monoresistant, chi-squared (1)=7.4 $p=0.007$; comparison MDR-TB (excluding XDR-TB) vs monoresistant, chi-squared $(1)=24.4$ p $<0.001$ ). Cases aged $\geq 65$ years were significantly more frequent among pan-susceptible cases $(17.3 \%$, p $<0.001)$ when compared to all other age groups (7.3-10.8\%).

Of the pan-susceptible TB cases, $28.1 \%$ were recorded with a foreign origin. While monoresistant cases were more frequently of foreign origin, $34.6 \%$ ( $p<0.001$ ), MDR-TB (excluding XDR-TB), and XDR-TB cases were 
TABLE 3

Rifampicin monoresistance, isoniazid monoresistance, and multidrug-resistant TB in EU/EEA countries, 2012

\begin{tabular}{|c|c|c|c|c|}
\hline Country & $\begin{array}{l}\text { Number of TB cases with } \\
\text { reported susceptibility } \\
\text { testing results to at least } \\
\text { isoniazid and rifampicin }\end{array}$ & $\begin{array}{l}\text { Rifampicin } \\
\text { monoresistant } \\
\mathrm{N}(\%)\end{array}$ & $\begin{array}{l}\text { Isoniazid } \\
\text { monoresistant } \\
\text { N (\%) }\end{array}$ & $\begin{array}{c}\text { MDR-TB } \\
\text { N (\%) }\end{array}$ \\
\hline Austria & 392 & $0(0.0)$ & $14(3.6)$ & $27(6.9)$ \\
\hline Belgium & 735 & $5(0.7)$ & $37(5.0)$ & $20(2.7)$ \\
\hline Bulgaria & 829 & $9(1.1)$ & $27(3.3)$ & $49(5.9)$ \\
\hline Cyprus & 49 & $\mathrm{o}(0.0)$ & $1(2.0)$ & $0(0.0)$ \\
\hline Czech Republic & 397 & $1(0.3)$ & $4(1.0)$ & $4(1.0)$ \\
\hline Denmark & 298 & $0(0.0)$ & $8(2.7)$ & $1(0.3)$ \\
\hline Estonia & 239 & $3(1.3)$ & $3(1.3)$ & $61(25.5)$ \\
\hline Finland & 222 & $0(0.0)$ & $8(3.6)$ & $3(1.4)$ \\
\hline France $^{a}$ & - & - & - & - \\
\hline Germany & 2,794 & $5(0.2)$ & $96(3.4)$ & $60(2.1)$ \\
\hline Greece $^{\mathrm{b}}$ & - & - & - & - \\
\hline Hungary & 449 & $2(0.4)$ & $17(3.8)$ & $11(2.4)$ \\
\hline Iceland & 5 & $0(0.0)$ & $\mathrm{o}(0.0)$ & $1(20.0)$ \\
\hline Ireland & 265 & $0(0.0)$ & $10(3.8)$ & $5(1.9)$ \\
\hline Italy ${ }^{a}$ & - & - & - & - \\
\hline Latvia & 766 & $0(0.0)$ & $28(3.7)$ & $106(13.8)$ \\
\hline Liechtenstein ${ }^{\mathrm{b}}$ & - & - & - & - \\
\hline Lithuania & 1,368 & $10(0.7)$ & $56(4.1)$ & $271(19.8)$ \\
\hline Luxembourg & 29 & $0(0.0)$ & $0(0.0)$ & $0(0.0)$ \\
\hline Malta & 14 & $0(0.0)$ & $0(0.0)$ & 0 (o.o) \\
\hline Netherlands & 656 & $1(0.2)$ & $23(3.5)$ & $11(1.7)$ \\
\hline Norway & 280 & $1(0.4)$ & $7(2.5)$ & $6(2.1)$ \\
\hline Poland & 4,659 & $9(0.2)$ & $104(2.2)$ & $31(0.7)$ \\
\hline Portugal & 1,321 & $1(0.1)$ & $21(1.6)$ & $17(1.3)$ \\
\hline Romania & 5,966 & $75(1.3)$ & $232(3.9)$ & $530(8.9)$ \\
\hline Slovakia & 181 & $0(0.0)$ & $4(2.2)$ & $1(0.6)$ \\
\hline Slovenia & 126 & $0(0.0)$ & $2(1.6)$ & $0(0.0)$ \\
\hline Spain ${ }^{\mathrm{a}}$ & - & - & - & - \\
\hline Sweden & 503 & $1(0.2)$ & $33(6.6)$ & $14(2.8)$ \\
\hline United Kingdom & 5,151 & $8(0.2)$ & $196(3.8)$ & $81(1.6)$ \\
\hline Total EU/EEA & 27,694 & $131(0.5)$ & $931(3.4)$ & $1,310(4.7)$ \\
\hline
\end{tabular}

EU/EEA: European Union/European Economic Area; MDR-TB: multidrug-resistant tuberculosis; TB: tuberculosis.

a No case-based reporting to the European Surveillance System (TESSy) of drug susceptibility testing results but aggregated results were reported to the World Health Organization's Tuberculosis Monitoring and Evaluation platform.

b No reporting to TESSy in 2012.

less frequently of foreign origin, respectively $18.7 \%$ and $12.5 \%$ (p<0.001 for both comparisons).

Previous treatment was reported for $14.4 \%$ of all laboratory-confirmed cases, but it was much more common in cases with MDR-TB (excluding XDR-TB), and XDR-TB, accounting for $53.1 \%$ ( $p<0.001$ ) and $77.3 \%$ ( $p<0.001)$, respectively compared to all other cases (pan-susceptible, monoresistant and polyresistant cases). Surprisingly, only $15.3 \%$ of the TB cases with a polyresistant pattern had previously received TB treatment. This is a bit more than the $11.9 \%(p=0.022)$ of the pan-susceptible that had previously received TB treatment, and comparable to the $18.0 \%(p=0.168)$ of monoresistant TB cases.

Fifteen countries reported case-based HIV testing results. Overall, only $28.1 \%$ of the TB cases had HIV status reported, but this percentage was much higher for MDR-TB (excluding XDR-TB), and XDR-TB cases, i.e. respectively $52.7 \%$ and $65.6 \%$. Also, the percentage testing positive for HIV was higher for MDR-TB (excluding XDR-TB) cases (10.4\%, pro.001), and XDR-TB cases ( $13.1 \%$, p $<0.001)$, when compared to all other cases 


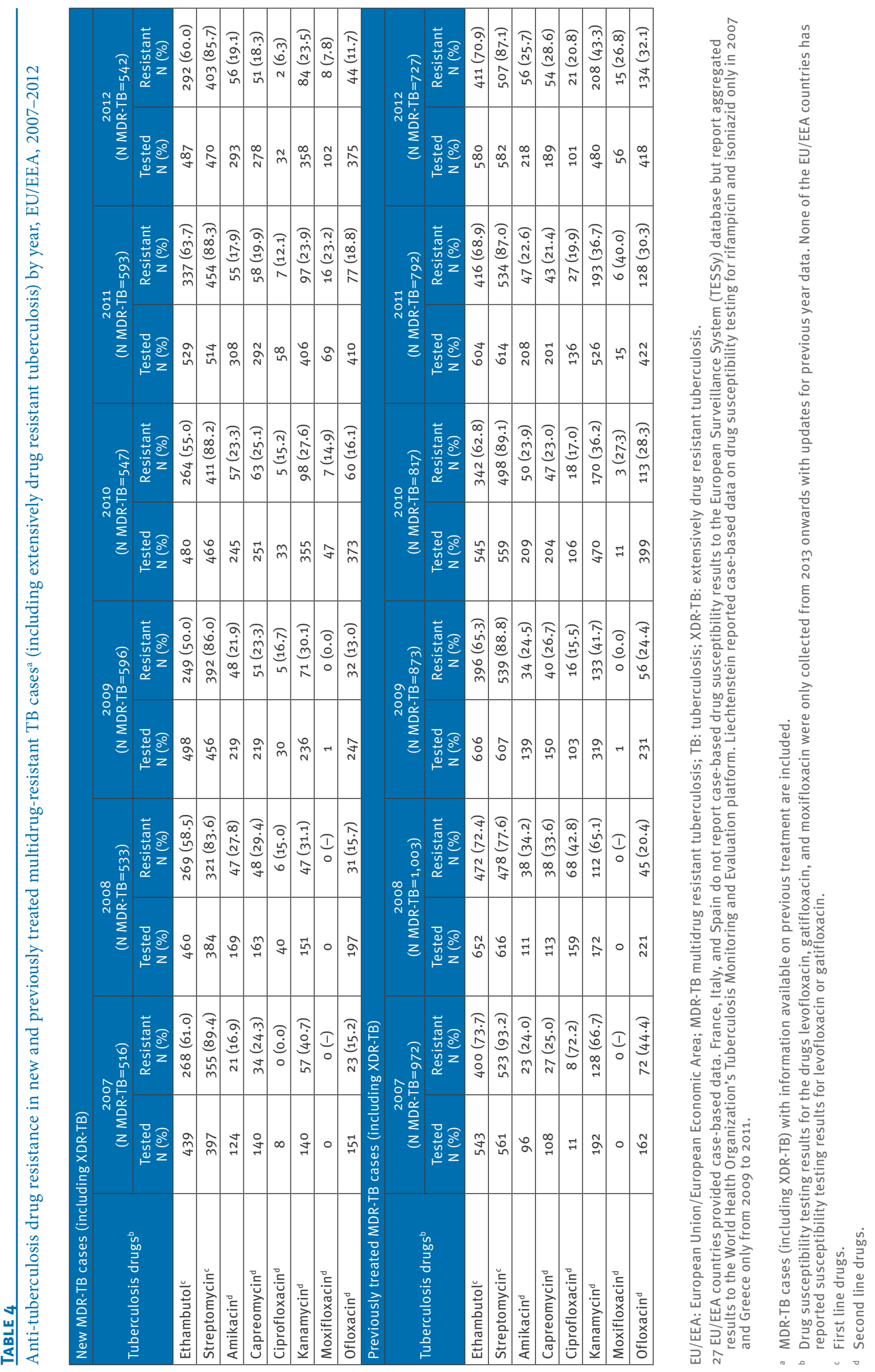




\section{TABLE 5}

Characteristics of all laboratory-confirmed TB cases tested for at least isoniazid and rifampicin resistance by drug resistance pattern, EU/EEA, 2012

\begin{tabular}{|c|c|c|c|c|c|c|}
\hline Characteristics & $\begin{array}{c}\text { Pan-susceptible } \\
\text { N (\%) }\end{array}$ & $\begin{array}{c}\text { Monoresistant } \\
\text { N (\%) }\end{array}$ & $\begin{array}{l}\text { Polyresistant } \\
\text { non-MDR-TB } \\
\text { N (\%) }\end{array}$ & $\begin{array}{c}\text { MDR-TB not } \\
\text { XDR-TB } \\
\text { N (\%) }\end{array}$ & $\begin{array}{l}\text { XDR-TB } \\
\text { N (\%) }\end{array}$ & $\begin{array}{l}\text { Total } \\
\text { N (\%) }\end{array}$ \\
\hline Total & $24,199(100.0)$ & $1,648(100.0)$ & $537(100.0)$ & $1,182(100.0)$ & $128(100.0)$ & $27,694(100.0)$ \\
\hline \multicolumn{7}{|l|}{ Sex } \\
\hline Female & $7,968(32.9)$ & $536(32.5)$ & $180(33.5)$ & $303(25.6)$ & $28(21.9)$ & $9,015(32.6)$ \\
\hline Male & $16,227(67.1)$ & $1,112(67.5)$ & $356(66.3)$ & $879(74.4)$ & $100(78.1)$ & $18,674(67.4)$ \\
\hline \multicolumn{7}{|l|}{ Age groups (years) } \\
\hline $0-14$ & $356(1.5)$ & $34(2.1)$ & $7(1.3)$ & $13(1.1)$ & $0(0.0)$ & $410(1.5)$ \\
\hline $15-24$ & $2,687(11.1)$ & $210(12.7)$ & $64(11.9)$ & $111(9.4)$ & $9(7.0)$ & $3,081(11.1)$ \\
\hline $25-44$ & $9,125(37.7)$ & $685(41.6)$ & $246(45.8)$ & $515(43.6)$ & $62(48.4)$ & $10,633(38.4)$ \\
\hline $45-64$ & $7,843(32.4)$ & $541(32.8)$ & $174(32.4)$ & $457(38.7)$ & $47(36.7)$ & $9,062(32.7)$ \\
\hline$\geq 65$ & $4,186(17.3)$ & $178(10.8)$ & $46(8.6)$ & $86(7.3)$ & $10(7.8)$ & $4,506(16.3)$ \\
\hline Unknown & $2(0.0)$ & $0(0.0)$ & $0(0.0)$ & $0(0.0)$ & $0(0.0)$ & $2(0.0)$ \\
\hline \multicolumn{7}{|l|}{ Origin } \\
\hline Foreign & $6,805(28.1)$ & $570(34.6)$ & $174(32.4)$ & $221(18.7)$ & $16(12.5)$ & $7,786(28.1)$ \\
\hline Native & $17,060(70.5)$ & $1,057(64.1)$ & $356(66.3)$ & $959(81.1)$ & $112(87.5)$ & $19,544(70.6)$ \\
\hline Unknown & $334(1.4)$ & $21(1.3)$ & $7(1.3)$ & $2(0.2)$ & $0(0.0)$ & $364(1.3)$ \\
\hline \multicolumn{7}{|l|}{ Previous treatment } \\
\hline No & $20,343(84.1)$ & $1,288(78.2)$ & $430(80.1)$ & $517(43.7)$ & $25(19.5)$ & $22,603(81.6)$ \\
\hline Yes & $2,885(11.9)$ & $296(18.0)$ & $82(15 \cdot 3)$ & $628(53.1)$ & $99(77 \cdot 3)$ & $3,990(14.4)$ \\
\hline Unknown & $971(4.0)$ & $64(3.9)$ & $25(4 \cdot 7)$ & $37(3.1)$ & $4(3.1)$ & $1,101(4.0)$ \\
\hline \multicolumn{7}{|l|}{ HIV status } \\
\hline HIV tested & $6,431(26.6)$ & $470(28.5)$ & $173(32.2)$ & $623(52.7)$ & $84(65.6)$ & $7,781(28.1)$ \\
\hline HIV infected ${ }^{a}$ & $282(4.4)$ & $41(8.7)$ & $15(8.7)$ & $65(10.4)$ & $11(13.1)$ & $414(5 \cdot 3)$ \\
\hline Pulmonary & $20,450(84.5)$ & $1,368(83.0)$ & $461(85.8)$ & $1,126(95 \cdot 3)$ & $127(99.2)$ & $23,532(85.0)$ \\
\hline Extra-pulmonary & $3,699(15 \cdot 3)$ & $280(17.0)$ & $75(14.0)$ & $55(4 \cdot 7)$ & $1(0.8)$ & $4,110(14.8)$ \\
\hline Unknown & $50(0.2)$ & $0(0.0)$ & $1(0.2)$ & $1(0.1)$ & $0(0.0)$ & $52(0.2)$ \\
\hline
\end{tabular}

EU/EEA: European Union/European Economic Area; HIV: human immunodeficiency virus; MDR-TB: multidrug resistant tuberculosis; TB: tuberculosis; XDR-TB: extensively drug-resistant tuberculosis.

27 EU/EEA countries provided case-based data. France, Italy, and Spain do not report case-based drug susceptibility results to the European Surveillance System (TESSy) database but report aggregated results to the World Health Organization's Tuberculosis Monitoring and Evaluation platform. Liechtenstein and Greece did not report to TESSy in 2012.

a The denominator for the calculation of percentage of HIV infected was the number of HIV-tested cases.

(pan-susceptible, monoresistant and polyresistant cases).

Over $95 \%$ of the MDR-TB (excluding XDR-TB), and XDR-TB cases had pulmonary TB. For the other resistance patterns, around $85 \%$ were reported to have pulmonary TB.

Treatment outcome of tuberculosis cases with drug resistance

In period from 2007 to $2011,77.9 \%$ of the pan-susceptible laboratory-confirmed TB cases with a test result for at least isoniazid and rifampicin resistance had a successful treatment outcome. Cases with a monoresistant or polyresistant non-MDR-TB drug resistance pattern showed slightly lower $(69.6 \%$ and $68.2 \%$, respectively) treatment success rates. For MDR (excluding XDR-TB) reported in period from 2007 to 2010 and XDR-TB cases reported in period between 2007 and 2009 , the treatment success rates were $32.2 \%$ and $19.1 \%$, respectively (Figure 3). Trends in successful treatment outcome were relatively stable over the years.

The treatment success rate of new pulmonary MDR-TB (excluding XDR-TB) cases was $48.2 \%$ and $21.4 \%$ for 
Successful treatment outcome of laboratory-confirmed tuberculosis (TB) cases tested for at least isoniazid and rifampicin resistance, by drug resistance pattern, EU/EEA, 2007-2011

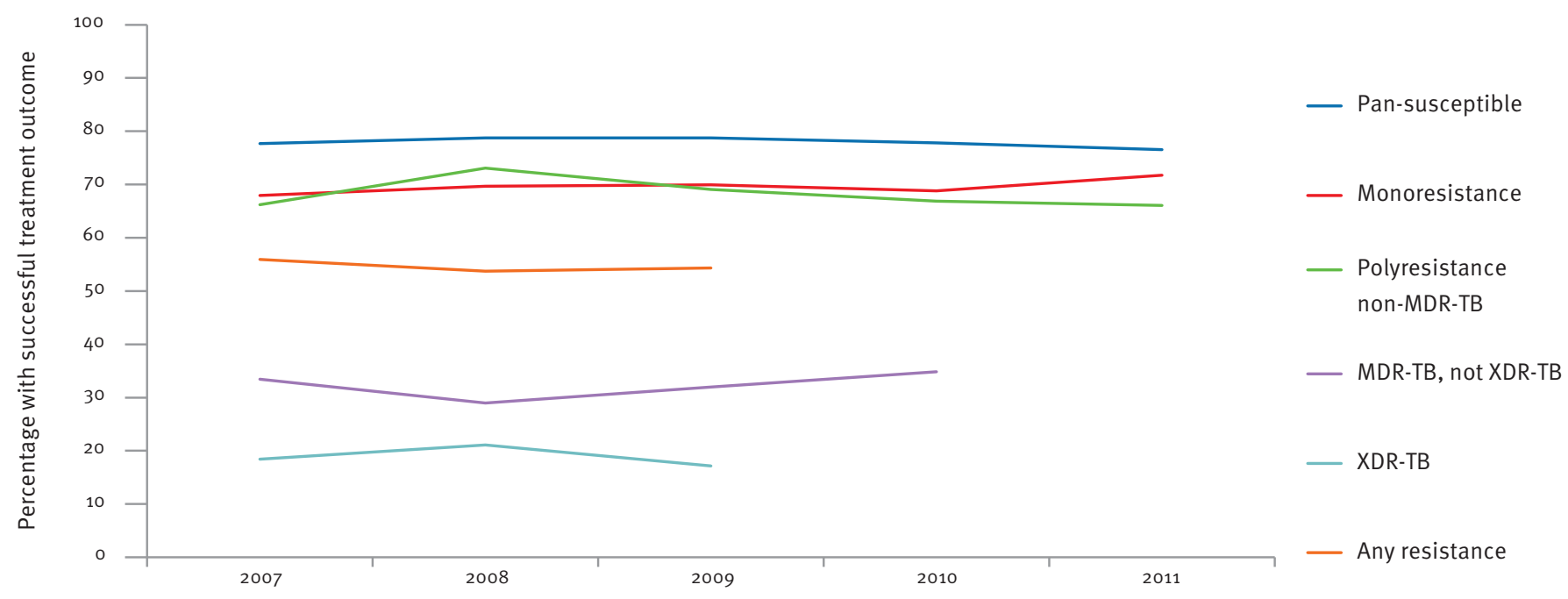

EU/EEA: European Union/European Economic Area.

27 EU/EEA countries provided case-based data. France, Italy, and Spain do not report case-based drug susceptibility results to the European Surveillance System (TESSy) database but report aggregated results to the World Health Organization's Tuberculosis Monitoring and Evaluation platform. Of the $27 \mathrm{EU} / \mathrm{EEA}$ countries included in the study, Liechtenstein reported case-based data on drug susceptibility testing for rifampicin and isoniazid only in 2007 and Greece only from 2009 to 2011.

previously treated pulmonary MDR (excluding XDR-TB) cases in the period between 2007 and 2010. For new pulmonary XDR-TB cases the treatment success rate was $49.2 \%$ in the period from 2007 to 2009 and for previously treated pulmonary XDR-TB cases it was $13.3 \%$.

Of all MDR-TB cases (including XDR-TB) diagnosed between 2007 and 2009, 32.4\% had a successful treatment outcome. Of all MDR-TB cases (including XDR-TB) that had an unsuccessful treatment outcome, $21.4 \%$ died, $24.5 \%$ failed treatment and $19.8 \%$ defaulted from treatment, $0.6 \%$ transferred out and for $1.4 \%$ outcome was unknown (Figure 4). Treatment outcomes did not show any improvement in the years 2007 to 2009 .

In period between 2007 and 2009, Romania reported a treatment success rate of $20.5 \%$ for 2,089 MDR-TB (including XDR-TB) cases. Since this significantly influences the overall picture for the EU/EEA, we provide treatment outcome results without the data reported by Romania. Without these data, $49.1 \%$ successfully finished treatment, $20.7 \%$ died, $7.5 \%$ failed treatment, $18.1 \%$ defaulted, $1.3 \%$ were transferred out, and $3.3 \%$ were reported as unknown.

\section{Discussion}

The percentage of laboratory-confirmed TB cases with different drug resistance patterns, i.e. pan-susceptible, mono-, and polydrug resistance, MDR-TB (excluding XDR-TB), and XDR-TB, has been stable for new cases during the period of the study, from 2007 to 2012. In new TB cases, drug resistance does not seem to be a significant problem with only $2 \%$ being diagnosed with MDR-TB. However, in previously treated TB cases, a much higher percentage, i.e. $16 \%$ is diagnosed with MDR-TB. The observed decline in the percentage of previously treated TB cases with MDR-TB from $22.3 \%$ in 2007 to $16.5 \%$ in 2010 can partly be explained by less selective testing. In $2007,37.2 \%$ of all previously treated TB cases were tested for isoniazid and rifampicin resistance and in 2010 this had increased to $46.6 \%$.

Our analysis also showed that treatment outcome results have been stable over the years with an acceptable treatment success rate in pan-susceptible TB cases, though below the target of $85 \%$ of the monitoring framework of the Framework Action Plan to Fight Tuberculosis in the European Union [9]. Measured against the set target of $70 \%$ in the monitoring framework of the Framework Action Plan to Fight Tuberculosis in the European Union [9], the treatment success rate of new pulmonary culture-positive MDR-TB was unacceptably low at only $48.2 \%$ in the period from 2007 to 2010.

Not all notified laboratory-confirmed TB cases had a result reported for rifampicin and isoniazid sensitivity testing. In 2010, the EU/EEA target for testing culture-confirmed TB cases for susceptibility to first-line TB drugs was set at $100 \%$ [9]. Even though a higher percentage of TB cases was tested in 2012 (78.5\%) 
compared to $2007(63.5 \%)$, the target was far from being reached for the EU/EEA overall. However, five EU/EEA countries have reached and nine are close to reaching the target, reporting DST results including for rifampicin and isoniazid for $95 \%$ or more of the laboratory-confirmed TB cases. The low percentage of notified laboratory-confirmed TB cases with a result reported for rifampicin and isoniazid drug sensitivity testing is mainly explained by the low percentage of testing results reported by Romania, the country that reported the highest number of laboratory-confirmed TB cases in the EU/EEA, and reported rifampicin and isoniazid DST results for less than half of their cases. In addition, several EU countries do not report casebased DST results to TESSy but report aggregated results to the WHO's Tuberculosis Monitoring and Evaluation platform.

Information on the drug susceptibility pattern for second-line TB drugs is likely to be beneficial for the treatment outcome of MDR-TB patients as drug susceptibility results allow for adequate choice of treatment. The EU/EEA surveillance data showed that in 2012, for $68.0 \%$ of MDR-TB cases, a testing result for susceptibility to second-line TB drugs was reported and between 2008 and 2010 there was a sharp increase. ECDC started collection of drug susceptibility results for second line drugs in 2008. Few countries provided second line drug susceptibility data for the years before 2008 . After 2008 the number of countries reporting second line drug susceptibility data increased and also the completeness of reporting improved.

TB patient characteristics differed across resistance patterns. Characteristics of cases with mono- and polydrug resistance were largely similar to those of pansusceptible cases whereas MDR- and XDR-TB cases seemed to differ from cases with other resistance patterns. Of the limited number of risk factors that we could evaluate, we found that male sex, native origin, previous TB treatment, and HIV infection were more frequent in MDR-TB (excluding XDR-TB) and XDR-TB. Of note is the low percentage of TB cases for which HIV status was reported. Other studies have shown similar results [17-21]. Especially a history of previous treatment is frequently identified as a strong risk factor for MDR-TB $[17,18,21]$. Other reported risk factors are a history of imprisonment, alcohol abuse, smoking, and hospitalisation for more than 14 days $[17,18,20]$.

Treatment outcome results of TB cases with a monoor polydrug resistance (not MDR-TB) were only slightly less favourable compared to those of pan-susceptible TB cases. The reported treatment success rates for MDR-TB and XDR-TB cases were significantly below the EU/EEA target of $70 \%$ [9]. In a recently published meta-analysis, using individual MDR-TB patient data, the pooled treatment success rate was $54 \%$ and ranged between 11 and $89 \%$ in the different studies that were included [22]. Surveillance data reported in the Global Tuberculosis Report 2012 showed that MDR-TB
FIGURE 4

Treatment outcome of all MDR-TB cases (including XDR-TB) after 36 months, EU/EEA countries ${ }^{\mathrm{a}}$, 2007-2009

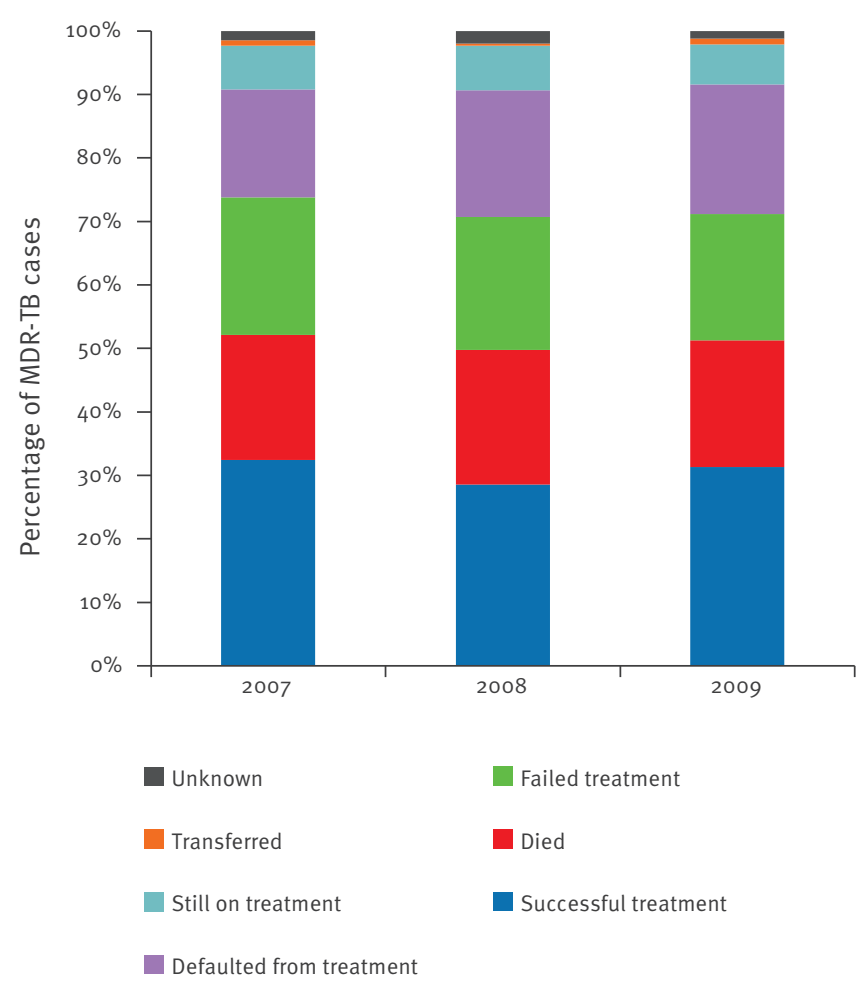

EU/EEA: European Union/European Economic Area; MDR-TB: multidrug resistant tuberculosis; XDR-TB: extensively drug resistant tuberculosis.

a Countries reporting treatment outcome for the different cohorts are provided in [27].

treatment success rates ranged between $44 \%$ and $58 \%$ for the 2009 treatment cohort in the different WHO regions [1]. Since $58.5 \%$ of the MDR-TB patients in the EU/EEA were notified by Romania between 2007 and 2009, the treatment outcomes achieved in this country have a considerable effect on the overall MDR-TB treatment outcomes in the EU/EEA.

In the analysis we included confirmed TB cases according to the EU case definition with available data on drug susceptibility for at least isoniazid and rifampicin. These inclusion criteria may potentially underestimate the percentage of TB cases with any resistance as well as polydrug resistance.

This study is based on the TB surveillance data submitted to ECDC by the EU/EEA countries. As listed above, this limits the information available on risk factors. Also, not all reported information is complete and the quality of the reported information is the responsibility of the individual country. However, the substantial amount of data available allows for obtaining a rather adequate picture of the drug resistance situation in the EU/EEA. 
The EU/EEA TB surveillance system does not contain information on drug susceptibility data for the drug pyrazinamide. This is because of the technical complexity of achieving reliable and reproducible results. Also, there is no external quality assurance for pyrazinamide available [23]. Pyrazinamide is important in the treatment of tuberculosis and is included in many of the new TB regimens that are currently evaluated [24].

\section{Conclusion and recommendations}

The available data show that the number of TB cases with drug resistance is stable in the EU/EEA, but not declining. Patients having drug resistant TB need to sustain longer treatment with more drugs, and they have worse treatment outcomes, especially if diagnosed with MDR-TB or XDR-TB. Also, treatment costs of MDR-TB are at least five times higher compared to treatment costs of drug susceptible TB [25]. Health systems in EU/EEA countries should be prepared to adequately diagnose and treat drug-resistant TB, and test all TB cases for drug susceptibility in a quality-assured laboratory [26]. Monitoring of drug resistance data at national and EU/EEA level should be continued to support identification of risk groups and areas where improvement may be needed.

\section{Conflict of interest}

None declared.

\section{Authors' contributions}

Marieke J. van der Werf designed and drafted the manuscript and coordinated the input from the other authors. Csaba Ködmön performed the data extraction and analysis and provided input for specific parts of the manuscript, reviewed the draft manuscript, and gave final approval of the version to be published. Vahur Hollo checked the data provided in the manuscript, reviewed the draft manuscript, and gave final approval of the version to be published. Andreas Sandgren checked the data provided in the manuscript, performed the statistical testing, reviewed the draft manuscript, and gave final approval of the version to be published. Phillip Zucs provided input for specific parts of the manuscript, reviewed the draft manuscript, and gave final approval of the version to be published.

\section{References}

1. World Health Organization. Global tuberculosis report 2012 Geneva: WHO; 2012.

2. European Centre for Disease Prevention and Control (ECDC)/ World Health Organization (WHO) Regional Office for Europe. Tuberculosis surveillance and monitoring in Europe, 2013. Stockholm: ECDC;2013.

3. Schwoebel V, Lambregts-van Weezenbeek CS, Moro ML, Drobniewski F, Hoffner SE, Raviglione MC, et al. Standardization of antituberculosis drug resistance surveillance in Europe. Recommendations of a World Health Organization (WHO) and International Union Against Tuberculosis and Lung Disease (IUATLD) Working Group. Eur Respir J. 2000;16(2):364-71.

4. Kodmon C, Hollo V, Huitric E, Amato-Gauci A, Manissero D. Multidrug- and extensively drug-resistant tuberculosis: a persistent problem in the European Union European Union and European Economic Area. Euro Surveill. 2010;15(11). pii: 19519.
5. Perdigão J, Macedo R, Silva C, Pinto C, Furtado C, Brum L, et al. Tuberculosis drug-resistance in Lisbon, Portugal: a 6-year overview. Clin Microbiol Infect. 2011;17(9):1397-402.

6. Papaventsis D, Nikolaou S, Karabela S, Ioannidis P, Konstantinidou E, Marinou I, et al. Tuberculosis in Greece: bacteriologically confirmed cases and anti-tuberculosis drug resistance, 1995-2009. Euro Surveill. 2010;15(28). pii: 19614 .

7. Kruijshaar ME, Watson JM, Drobniewski F, Anderson C, Brown TJ, Magee JG, et al. Increasing antituberculosis drug resistance in the United Kingdom: analysis of National Surveillance Data. BMJ. 2008;336(7655):1231-4. http://dx.doi.org/10.1136/ bmj.39546.573067.25

8. Alberte-Castineiras A, Campos-Bueno A, Lopez-Urrutia L, Alvarez-Alonso E, Megias G, Ojeda-Fernandez E, et al. Resistencias a farmacos de Mycobacterium tuberculosis en la Comunidad de Castilla y Leon (Espana), 2001-2005: tercer estudio multicentrico. [Drug-resistance in Mycobacterium tuberculosis in Castilla y Leon, Spain, 2001-2005: third collaborative study]. Enferm Infecc Microbiol Clin. 2010;28(10):706-9. Spanish. http://dx.doi.org/10.1016/j. eimc.2010.02.011

9. European Centre for Disease Prevention and Control (ECDC). Progressing towards TB elimination. A follow-up to the Framework Action Plan to Fight Tuberculosis in the European Union. Stockholm: ECDC; 2010.

10. Maguire H, Brailsford S, Carless J, Yates M, Altass L, Yates $S$, et al. Large outbreak of isoniazid-monoresistant tuberculosis in London, 1995 to 2006: case-control study and recommendations. Euro Surveill. 2011;16(13).pii=19830.

11. Dalton T, Cegielski P, Akksilp S, Asencios L, Campos Caoili J, Cho SN, et al. Prevalence of and risk factors for resistance to second-line drugs in people with multidrug-resistant tuberculosis in eight countries: a prospective cohort study. Lancet. 2012;380(9851):1406-17. http://dx.doi.org/10.1016/ S0140-6736(12)60734-X

12. Ditah IC, Reacher M, Palmer C, Watson JM, Innes J, Kruijshaar $\mathrm{ME}$, et al. Monitoring tuberculosis treatment outcome: analysis of national surveillance data from a clinical perspective. Thorax. 2008;63(5):440-6. http://dx.doi.org/10.1136/ thx.2006.073916

13. Farah MG, Tverdal A, Steen TW, Heldal E, Brantsaeter AB, Bjune $\mathrm{G}$. Treatment outcome of new culture positive pulmonary tuberculosis in Norway. BMC Public Health. 2005;5:14. http:// dx.doi.org/10.1186/1471-2458-5-14

14. Diel R, Niemann S. Outcome of tuberculosis treatment in Hamburg: a survey, 1997-2001. Int J Tuberc Lung Dis. 2003;7(2):124-31.

15. Wang CS, Yang CJ, Chen HC, Chuang SH, Chong IW, Hwang $\mathrm{J}$, et al. Impact of type 2 diabetes on manifestations and treatment outcome of pulmonary tuberculosis. Epidemiol Infect. 2009;137(2):203-10. http://dx.doi.org/10.1017/ So950268808000782

16. European Commission. Commission Decision of 28 April 2008 amending Decision 2002/253/EC laying down case definitions for reporting communicable diseases to the Community network under Decision No 2119/98/EC of the European Parliament and of the Council (notified under document number C (2008) 1589). Official Journal of the European Union. Luxembourg: Publications Office of the European Union.18.06.2008:L156/46. Available from: http://eur-lex. europa.eu/LexUriServ/LexUriServ.do?uri=0J:L:2008:159:0046 :0090:EN:PDF

17. Skrahina A, Hurevich H, Zalutskaya A, Sahalchyk E, Astrauko A, Hoffner S, et al. Multidrug-resistant tuberculosis in Belarus: the size of the problem and associated risk factors. Bull World Health Organ. 2013;91(1):36-45. http://dx.doi.org/10.2471/ BLT.12.104588

18. Andrews JR, Shah NS, Weissman D, Moll AP, Friedland G, Gandhi NR. Predictors of multidrug- and extensively drugresistant tuberculosis in a high HIV prevalence community. PLoS One. 2010;5(12):e15735. http://dx.doi.org/10.1371/ journal.pone.0015735

19. Faustini A, Hall AJ, Perucci CA. Risk factors for multidrug resistant tuberculosis in Europe: a systematic review. Thorax. 2006;61(2):158-63. http://dx.doi.org/10.1136/thx.2005.045963

20. Ruddy M, Balabanova Y, Graham C, Fedorin I, Malomanova $\mathrm{N}$, Elisarova $\mathrm{E}$, et al. Rates of drug resistance and risk factor analysis in civilian and prison patients with tuberculosis in Samara Region, Russia. Thorax. 2005;60(2):130-5. http:// dx.doi.org/10.1136/thx.2004.026922

21. de Souza MB, Antunes CM, Garcia GF. Multidrug-resistant Mycobacterium tuberculosis at a referral center for infectious diseases in the state of Minas Gerais, Brazil: sensitivity profile and related risk factors. J Bras Pneumol. 2006;32(5):430-7.

22. Ahuja SD, Ashkin D, Avendano M, Banerjee R, Bauer M, Bayona JN, et al. Multidrug resistant pulmonary tuberculosis 
treatment regimens and patient outcomes: an individual patient data meta-analysis of 9,153 patients. PLoS Med. 2012;9(8):e1001300. http://dx.doi.org/10.1371/journal. pmed.1001300

23. Drobniewski F, Rusch-Gerdes S, Hoffner S; Subcommittee on Antimicrobial Susceptibility Testing of Mycobacterium tuberculosis of the European Committee for Antimicrobial Susceptibility Testing of the European Society of Clinical Microbiology and Infectious Diseases (ESCMID). Antimicrobial susceptibility testing of Mycobacterium tuberculosis (EUCAST document E.DEF 8.1)--report of the Subcommittee on Antimicrobial Susceptibility Testing of Mycobacterium tuberculosis of the European Committee for Antimicrobial Susceptibility Testing (EUCAST) of the European Society of Clinical Microbiology and Infectious Diseases (ESCMID) Clin Microbiol Infect. 2007;13(12):1144-56. http://dx.doi. org/10.1111/j.1469-0691.2007.01813.x

24. TB Alliance. [Accessed 28 Jan 2014]. Available from: http:// www.tballiance.org/downloads/Pipeline/TBA\%20Pipeline $\% 20$ Q1\%202014(2).pdf

25. Diel R, Vandeputte J, de Vries G, Stillo J, Wanlin M, Nienhaus A. Costs of tuberculosis disease in the European Union: a systematic analysis and cost calculation. Eur Respir J. 2014;43(2):554-65. http://dx.doi. org/10.1183/09031936.00079413

26. Migliori GB, Zellweger JP, Abubakar I, Ibraim E, Caminero JA De Vries G, et al. European union standards for tuberculosis care. Eur Respir J. 2012;39(4):807-19. http://dx.doi. org/10.1183/09031936.00203811

27. European Centre for Disease Prevention and Control (ECDC)/ World Health Organization (WHO) Regional Office for Europe. Tuberculosis surveillance and monitoring in Europe, 2014. Stockholm: ECDC;2014. 